\title{
El sentido de lo público en el trabajo a prueba de la restructuración productiva: el caso de los centros de llamadas
}

\author{
The meaning of 'public utility' at work- \\ place against the productive restructur- \\ ing: a case study in call centers
}

${ }^{1}$ Doutor em Sociologia. Miembro del laboratorio Genre, Travail et Mobilités (GTM) Université Lille 1, France.

\begin{abstract}
Resumen
En los centros de llamadas, y más generalmente como tendencia que afecta a la globalidad del mundo del trabajo desde hace unas décadas, los empleadores no parecen sólo obsesionados por la necesidad de objetivar los tiempos y los gestos del trabajo sino que parecen dar cada vez más importancia a la implicación del asalariado, a su subjetividad, para que haga un uso de su tiempo de trabajo y de sus competencias lo mas eficaz posible desde el punto de vista de la organización. Si la subjetividad entra en el punto de mira de la organización, se puede suponer que la rivalidad se desplaza también a ese terreno, al del uso subjetivo del tiempo del trabajo. Para el desarrollo de esta doble hipótesis nos apoyamos en los resultados de una investigación por inmersión que realizamos en el seno de una plataforma telefónica.
\end{abstract}

Palabras claves: control, resistencia, subjetividad, implicación, seducción, telemarketing, trabajo emocional.

\begin{abstract}
In call centers, and more generally as a tendency affecting the whole work world since a few decades on, employers seem not just obsessed about the need of improving work times and work action, but they also seem to give more importance to the employee implication and his/her subjectivity, in order to make the worker use of his/her working time and abilities in the most efficient way from the point of view of the company. When personal subjectivity becomes a target for the company, we can suppose that also rivalry moves to the field of the subjectivity working time. In order to develop this double assumption, we base our article on some conclusions obtained from an immersion research fulfilled in a telephone platform.
\end{abstract}

Keywords: control, resistance, subjectivity, implication, seduction, telemarketing, emotional work. 


\section{Los centros de llamada: un paradigma de las organizaciones modernas}

En el curso de las dos últimas décadas, el desarrollo exponencial de patologías profesionales como el estrés, el acoso moral y el sufrimiento en el trabajo ha despertado un interés creciente entre los estudiosos del mundo del trabajo y los profesionales de la salud, y en consecuencia un replanteamiento profundo de la cuestión y de la manera de abordarla. En efecto, si en el periodo anterior taylorista el sufrimiento psíquico estaba más bien ligado a la división en clase de la sociedad, y más particularmente al poder limitado de acción, desde el punto de vista ontológico, de asalariados despojados de la capacidad de expresar su propio punto de vista en el trabajo, hoy estamos más bien confrontados a una difusión al conjunto del mundo del trabajo de dichos fenómenos de sufrimiento psíquico y de hastío en el trabajo².

Estos elementos abren la vía a una serie de cuestionamientos genéricos que deberemos replantear para el caso específico de los centros de llamadas: ¿qué tipo de mecanismos provocan que las formas de organización del trabajo actual sean peligrosas para la identidad y para la salud mental de los trabajadores? ¿Cómo es posible que estas formas se extiendan incluso a las categorías de trabajadores considerados anteriormente como privilegiados, como es el caso de los trabajadores de oficina (los trabajadores de cuello blanco) o los cuadros intermedios y superiores de la empresa?

Responder a estas preguntas requiere un análisis pormenorizado de la actividad, pensamos, desde la perspectiva de la sociología del trabajo. Por ejemplo, si miramos detenidamente el panorama de las evoluciones actuales de la organización del trabajo, éstas aparecen en efecto como portadoras de transformaciones que modifican la relación de los trabajadores con el trabajo, y esto independientemente del sector de actividad ocupado y de la categoría socioprofesional de origen.

Remontémonos al taylorismo: la situación de trabajo dejaba poco lugar para la improvisación. La industria producía en masa productos estandardizados para mercados de consumo de primer nivel; y el público que descubría el consumo compraba lo que se le proponía.
La actividad de producción era enteramente previsible. El organizador podía pretender prescribir detalladamente las actividades de trabajo y tratar a los asalariados como simples ejecutores. Por otro lado, las rivalidades de clase se organizaron, en la unidad de producción, en torno al uso objetivo (o si se prefiere intensivo) del tiempo de trabajo, ya que el tiempo subjetivo en modo alguno era objeto de discusión: simplemente era negado por parte de la ingeniería de producción.

Este cuadro ideal, y sus figuras míticas de referencia (entre ellas el famoso "gorila domesticado" del ingeniero Taylor) se han transformado radicalmente. La gestión en flujo sustituye a las modalidades anteriores de organización (DURAND, 2004) y la actividad depende cada vez más de la presión directa del cliente, del paciente o del usuario $^{3}$. La fe ingenieril en la racionalización completa de los medios se desmorona estrepitosamente ya que el fin depende cada vez más de la capacidad de adaptación sistemática por parte de los trabajadores a las variaciones, a los aleas, a los condicionantes imprevistos de producción que pueden aparecer inevitablemente en cualquier fase del proceso productivo. La llamada a la autonomía, a la iniciativa, a la responsabilidad de los trabajadores, proviene inmediatamente de esta incapacidad de los ingenieros por prescribir completamente las actividades de trabajo (FLICHY \& ZARIFIAN, 2003). Las condiciones de valorización del capital de la empresa tienden a ser descentralizadas, en el acto mismo de trabajo y a través de la interiorización subjetiva por parte de los trabajadores de su responsabilidad colectiva en dicho proceso de valorización (LINHART \& MOUTET, 2005). Resultado incuestionable: las evoluciones de la organización del trabajo requieren cada vez más la integración de la inteligencia y de la personalidad de los trabajadores en el proceso de trabajo.

Se podría pues suponer a simple vista que dichas evoluciones son positivas en regla general para el conjunto de los trabajadores, en el sentido que el significativo incremento de su poder de acción sobre el trabajo puede haber supuesto una mejora sustancial de las condiciones de salud mental de los trabajadores. Sin embargo, como decíamos anteriormente, las expec- 
tativas distan mucho de los resultados concretos contrastables. Es por tanto conveniente y necesario tratar de comprender cómo las perspectivas de autovalorización propuestas por las actuales organizaciones del trabajo pueden transformar este hipotético desarrollo libre de las propias capacidades cognitivas de los asalariados en una auténtica pesadilla para un número considerable de trabajadores.

Para desmenuzar esta paradoja, recurrimos a algunos resultados de una investigación que desarrollamos, una inmersión de cuatro meses de duración con los trabajadores y trabajadoras de una plataforma telefónica del sector de la distribución energética (PHONING), complementada con entrevistas en profundidad a cuadros, responsables de formación, sindicalistas y teleoperadores del centro. La elección de un centro de llamadas para este estudio no es baladí. Los centros de llamadas parecen en efecto un espacio paradigmatico de las evoluciones que hemos referido, donde los organizadores de la producción no parecen sólo obsesionados por la necesidad de objetivar los tiempos y los gestos del trabajo sino que parecen dar cada vez más importancia a la implicación del asalariado, a su subjetividad, para que haga un uso de su tiempo de trabajo y de sus competencias lo mas eficaz posible, aunque siempre, y este punto es fundamental, desde el punto de vista de los objetivos de la empresa.

Como veremos en el estudio de caso propiamente dicho, la llamada a la implicación de los trabajadores se acompaña en nuestra plataforma telefónica de una serie de dispositivos de control de tipo normativo, que buscan la estandardización y racionalización del comportamiento necesario en el trabajo, de la subjetividad de cada individuo, a partir de un modelo determinado de lo que es "ser un buen consejero”. Dichos mecanismos serán entendidos como práctica de control, abierta por tanto a la reinterpretación y a la resistencia por parte de los trabajadores aunque siempre dentro de los límites configurados por los propios dispositivos. Por ello, pondremos la atención en la conflictividad articulada en torno a los usos (intensidad y cualidad) a menudo contradictorios de la subjetividad en las actividades de trabajo en los centros de llamadas.

Consideraremos que si la subjetividad entra en el punto de mira de la organización, se puede suponer que la rivalidad (y el sufrimiento) se desplazan también a ese terreno, al del uso subjetivo del tiempo del trabajo como afirmación práctica de un imperativo de nuevo cuño. Como bien escribe Ricardo Antunes (2005, p. 132):

como a alienação é a produção do capital realizada pelo trabalho, ela deve ser entendida como atividade, estando sempre em disputa (...) O processo de alienação é, portanto, vivenciado cotidianamente pelo trabalho, e a desalienação é parte imprescindível desse processo.

En el mismo sentido que el investigador brasileño, sostenemos la hipótesis que el uso ético del autocontrol de los trabajadores, de la implicación en el desempeño de su actividad, puede estar a la base de colectivos de trabajo renovados.

Organizamos el texto como sigue. En primer lugar, presentamos brevemente algunos datos relativos a la evolución del sector de actividad y a las características genéricas de la mano de obra y de los procesos de selección y reclutamiento de los trabajadores insertos en dichos procesos de trabajo, de modo a componer un marco general de comprensión del fenómeno. En segundo lugar, volveremos sobre los dos aspectos que componen la actividad de los teleconsejeros, el aspecto cuantitativo y el aspecto cualitativo, tomando en consideración los dispositivos desplegados por la dirección del centro para garantizar la producción eficaz del servicio propuesto. Pensamos que la racionalización del proceso de producción de servicios es insuficiente en un contexto productivo donde la manera de trabajar se identifica de modo inmediato con la calidad del producto. Pensamos que toda la paradoja organizacional de los centros de llamadas está aquí presente y reposa sobre las espaldas de los teleoperadores en cada situación de trabajo. Por último, demostraremos cómo en un contexto de normativización de los comportamientos, el control de la subjetividad deviene terreno de contestación de racionalidades contradictorias, y puede situarse a la base de la conformación de colectivos de trabajo renovados. Así, la capacidad de los trabajadores para implicarse en la resolución de los problemas del cliente, contraviniendo incluso en ocasiones las directivas de la gerencia en este sentido, permite la conformación de espacios críticos alejados de la racionalidad empresarial, y que como veremos pueden dar lugar, como de hecho sucedió, a un nuevo tipo de movilización colectiva de carácter bastante novedoso. 
${ }^{4}$ Ver al respecto Les centres d'appel: un secteur en clair-obscur de Delaunay, J.-C. (2004), fruto de un trabajo colectivo en el seno del programa internacional TOSCA de investigación articulado en torno a la emergencia de los centros de llamadas, en el que participamos una decena de investigadores del lado francés.

\section{Desarrollo y características principales de los centros de llamadas en Francia}

Los centros de llamada representan una nueva e importante forma de trabajo en lo que respecta, en primer lugar, a la evolución del sector de actividad. Cada vez más empresas recurren a este tipo de dispositivos y le otorgan un lugar preponderante, central, en sus respectivas estrategias comerciales. En Francia, el número de empleados se ha multiplicado por cuatro entre 1996 y 2003, pasando de 60.000 a 250.000 empleos (fuentes CFDT), con un crecimiento anual de entre el $20 \%$ y el 30\% (LECHAT \& DELAUNAY, 2003). A nivel europeo, el número de trabajadores en centros de llamadas supera ampliamente el millón (DUFAU \& STUCHLIK, 2002). Estos números, ya de por sí extraordinarios, podrían verse incrementados si tomamos en consideración el interés creciente de las colectividades y servicios públicos por la puesta en aplicación de dichos dispositivos. Algunas estimaciones consideran que, en algunos años, el uno por ciento de la mano de obra europea estará empleada en algún tipo de centro de llamadas (fuentes Datamonitor).

Las empresas que recurren a este tipo de dispositivo lo califican habitualmente de "centro de relación con la clientela"; a través de estos centros, se suelen reagrupar la mayoría de los servicios propuestos a los clientes, ya sea de venta, de prospección de mercados, de información o de servicio post-venta de atención al consumidor. El cliente, omnipresente en los discursos de la empresa contemporánea, es a la vez principio de organización y principio de legitimación de las nuevas formas de trabajo en los centros de llamada. Es por ejemplo en nombre del cliente que la jerarquía ejerce sus presiones sobre los asalariados. Al tiempo, la empresa debe ser cada vez más capaz de acercarse del cliente, de tomar en cuenta sus necesidades, sus aspiraciones, de poder proponerle cada vez nuevos productos y nuevos servicios. El crecimiento exponencial de los centros de llamadas refleja entonces la transformación de los discursos, de los comportamientos, de las organizaciones de trabajo que se reconstituyen por y para el cliente en una dinámica de conquista y fidelización comercial continuas. Boltansky y Chiapello (1999) consideran precisamente estas dinámicas como uno de los principios fundamentales de lo que estos autores llaman "el nuevo espíritu del capitalismo".

En paralelo, la penetración del mercado dentro de las organizaciones obliga a éstas a someterse al imperativo de la reactividad, a buscar cada vez más nuevos espacios de relación directa y continua con el mercado, por lo que el dispositivo económico en su conjunto podría ser calificado de "espiral comercial sin límites".

En términos de características de la mano de obra, cabe señalar que los centros de llamada son un sector de actividad mayoritariamente joven y fuertemente femininizado. ¿Cuáles han sido los criterios que han regulado la selección empresarial de mano de obra en los centros de llamadas? Según la encuesta TOSCA ${ }^{4}$ que realizamos en 2002, 64\% de la fuerza de trabajo empleada en los centros de llamadas es mano de obra femenina, contra un 36\% masculina. Por edades, cabe destacar que el segmento más representado es el de 25-35 años, con un $49 \%$ del total de empleados (tabla 1).

Sorprendentemente, la mayor parte de los trabajadores del muestreo TOSCA tiene una antiguedad de más de cinco años (69\%), lo que indica que hay una continuidad en el empleo de los centros de llamadas mucho mayor de lo que se supone. En este sentido, un $90 \%$ de los trabajadores muestreados dispone de un contrato fijo, y sólo un $10 \%$ restante trabaja con contrato temporal.
Tabla 1 Personal en los centros de llamada por categoría de edad (\%) y por sexo, en Francia

\begin{tabular}{lcccc}
\hline Edad/sexo & $<25$ años & 25-35 años & 35-50 años & $>50$ años \\
\hline Hombres & 18.8 & 37.5 & 31.2 & 12.5 \\
Mujeres & 14.8 & 55.6 & 22.2 & 7.4 \\
Total & 16.3 & 48.8 & 25.6 & 9.3 \\
\hline
\end{tabular}

Fuente: Tosca (2002) 
Nuestra encuesta muestra también que la formación inicial no juega un papel determinante en la selección de mano de obra. Los criterios de dicha selección se construyen a partir de los puestos de trabajo y a menudo están relacionados con las características individuales de los candidatos, no identificables a partir del criterio relativo a la educación formal. Sin embargo, cabe señalar que el nivel de formación general es más bien elevado, aunque no tanto como afirma la retórica de las direcciones de las empresas que dicen preferir trabajadores altamente cualificados. El estudio TOSCA muestra que más de $2 / 3$ de la población total dispone al menos del bachillerato. En regla general, los hombres tienen un nivel de formación superior al de las mujeres.

En cuanto a los procesos de reclutamiento, la trilogía "curriculum, entrevista de trabajo y recomendaciones" es decisiva en dichos proceso. En particular, y aquí nos referimos a los datos obtenidos en nuestro trabajo de campo en PHONING, la empresa requiere una serie de referencias que le permitan efectuar una preevaluación de las actitudes y las capacidades de los candidatos al empleo. Ninguna capacitación técnica particular o formativa es requerida. Como nos indicaba un responsable del proceso de reclutamiento:

Buscamos esencialmente la esencia de la persona, lo demás lo aprenderán más tarde, estamos hablando de gente de veintitantos años, por tanto todo lo demás lo aprenderán con el tiempo, porque las tecnologías cambiarán, porque no hay nada definitivo... Queremos que tengan una buena actitud, que estén motivados, es eso lo que retenemos en prioridad, no nos interesa particularmente que estén cualificados. (Responsable del proceso de selección, PHONING)

Aquí buscamos personas sólidas, física y psicológicamente, porque trabajar seis o siete horas al teléfono es muy fatigoso... en muchos centros de llamadas los operadores hacen cinco o seis años en esta profesión y luego se van a otra parte, y son jóvenes...aquí reclutamos personal que en principio va a quedarse más años, diez o quince, así que necesitamos que tengan esa solidez física y mental. (Cuadro superior $P H O N I N G$ )
Conviene en primer lugar pues señalar el lugar preponderante acordado a las denominadas "competencias sociales" desde los procesos mismos de reclutamiento en los centros de llamadas como ya demostraron para el caso escocés Callaghan y Thompson (2002). La solidez física y psicológica y, por supuesto, la motivación, y otros aspectos como la personalidad del candidato al empleo, su energia, el entusiasmo supuesto, el "feeling", las habilidades comunicacionales (el tono, el sentido de la discusión) son elementos caracteriales particularmente solicitados por parte de los responsables de dichos procesos de selección.

Señalemos al tiempo que el hecho de hablar en términos de "cualidades" más que en términos de "cualificación" tampoco es neutro: las jóvenes trabajadoras que serán empleadas en los centros de llamadas no son "poco cualificadas" porque hayan sido poco formadas por la institución escolar, por ejemplo, sino porque han sido muy bien formadas por el conjunto de trabajo productivo en situación de precariedad y también reproductivo que han desarrollado hasta el momento, y en esta dirección están perfectamente adaptadas a los empleos que se les propone. En este sentido, algunos autores han señalado igualmente una transformación de las formas de gestión de la mano de obra en función de dichas competencias sociales en una tendencia hacia la "psicologización de las relaciones sociales" dentro de los centros de llamadas (BUSCATTO, 2002).

Como vemos, el perfil típico reclutado se aproxima considerablemente a la imagen prototípica de la "amable señorita que sonríe" que aparece sistemáticamente en todas las campañas de comercialización de los centros de llamadas y de la que se sorprende Angel Lara (2004) en su artículo sobre los centros de llamadas españoles. Sin embargo, como demostramos a continuación, dichas campañas no sirven sólo para esconder la negatividad de las condiciones de trabajo en los centros de llamadas, sino que para los trabajadores insertos en dichos procesos de trabajo, la imagen de la "amable señorita que sonríe" se presenta como una verdadera norma de trabajo. 


\section{Las nuevas figuras profesionales en los centros de llamadas: del "gorila amaestrado" al "robot de seducción"}

Los centros de llamada representan una nueva e importante forma en lo que respecta a las evoluciones observadas en el proceso de trabajo. Algunos autores han señalado la diversidad de las situaciones de trabajo observables en los centros de llamadas a pesar de estar fundados en un mismo principio tecnológico (BUSCATTO, 2002), en función por ejemplo del sector de actividad, del objetivo de la operación, de su situación jurídica con respecto a la empresa nodriza (COSSALTER, 2005), de la talla de cada centro, de las políticas de gestión de mano de obra o incluso del tipo de implantación sindical (LECHAT \& DELAUNAY, 2003). Sin embargo, la emergencia y el desarrollo de los centros de llamadas parecen inscribirse en un proceso global de racionalización del trabajo de servicio, y este elemento parece ser común al conjunto de las situaciones de trabajo que podamos observar más allá de su propia diversidad.

Tomar en cuenta la intensa racionalización tecnológica, gestionaria y organizacional de los centros de trabajo para aprehender las actividades de trabajo y en consecuencia la salud mental de los trabajadores es, pensamos, un buen punto de partida, a condición que dicha perspectiva no ponga en tela de juicio justamente la especificidad del trabajo de servicio en relación a las tareas industriales, esto es, la dimensión interactiva de la relación de servicio (GADREY, 1996).

Por supuesto, la relación telefónica de las empresas con su clientela se encuentra frecuentemente marcada por una voluntad de control estricto de las interacciones telefónicas, tanto en su contenido como en su ritmo. Esta voluntad de control pasa a menudo, y con mayor o menor intensidad según los centros de llamadas, por una racionalización bastante sorprendente del intercambio verbal y de su tiempo. Desde el punto de vista del empleado afectado por estos procesos, dicha racionalización debe permitirle dirigir la conversación y controlar estrictamente su duración. El objetivo de dicha racionalización, guiada por criterios de rentabilidad, no es otro pues que conseguir que el trabajador de los centros de llamadas responda al mayor número de llamadas, en el menor tiempo posible, y todo ello respetando al máximo los contenidos prescritos del intercambio.
Los dispositivos "taylorizadores" de la actividad se refieren fundamentalemente a un aspecto de la actividad, el aspecto cuantitativo, que es prioritario en situación de trabajo. Sin embargo, los mismos trabajadores deben estar en medida de implicarse ni que sea mínimamente en la actividad, de dar al menos una cierta sensación de que se escucha al cliente, de adaptar las modalidades de conducción de la discusión al tipo de cliente y a sus estados de ánimo, del cliente y a los propios.

Este aspecto cualitativo de la actividad es fundamental para entender el deslizamiento que se opera en los centros de llamadas y que los distingue sustancialmente, desde el punto de vista subjetivo, de las condiciones de realización del trabajo industrial.

Desde el punto de vista de la gerencia, las razones para buscar una mínima implicación por parte del operador parecen obvias: en primer lugar porque mantener a un cliente cuesta menos caro que conseguir a otro. En segundo lugar, porque una llamada bien respondida puede permitir transformar un requerimiento de información en la venta de un servicio.

Desde el punto de vista de la mano de obra implicada en los procesos de trabajo, porque la subjetividad del trabajador, su actitud, sus emociones, son indisociables del trabajo y del producto, aunque no sea más que porque es preciso imprimir a la conversación el tono apropiado para cada situación.

Sin embargo, los registros que motivan a unos y otros a implicarse en el desarrollo de la actividad no tienen por qué ser coincidentes. Desde la perspectiva de la empresa, esto quiere decir que los trabajadores deben movilizar algunas de sus competencias "sociales" constituídas fundamentalmente en el espacio privado, pero que sin embargo dicha movilización no puede producirse de cualquier manera. La hipótesis que sostenemos es que, en los centros de llamadas, dispositivos de "entrenamiento" como el coaching, disfrazados de dispositivos formativos, sirven en realidad para abordar aspectos específicos del trabajo no completamente determinables por las reglas y las estructuras de control propias al trabajo rutinario. 
En los centros de llamadas se asiste así a la combinación inédita de modalidades de control, unas más tradicionales que atienden al aspecto cuantitativo de la actividad, las otras, normativas, que buscan racionalizar el tipo de comportamiento deseado a partir de un perfil tipo (el comercial seductor), y que provocan, en su articulación, una extensión pragmática del objeto de control.

Un trabajo bajo fuerte dependencia tecnológica

El trabajo en los centros de llamadas es un trabajo esencialmente de ejecución. El control jerárquico se efectúa fundamentalemente a través del sistema informático, por mediación de un software que posibilita la grabación de informaciones muy precisas que describen los resultados individuales y colectivos de la plataforma. Este mismo programa permite al supervisor, quien toma bajo su responsabilidad a un pequeño número de teleoperadores, la conexión y el seguimiento en tiempo real de los resultados de cualquier puesto de la plataforma: número de llamadas realizadas o recibidas, número de ventas o de contactos realizados, duración de las llamadas o del tiempo de pausa de cada asalariado...

Ni qué decir tiene que la transparencia de este tipo de control es prácticamente total, permitiendo la puesta en aplicación de dispositivos de gestión que pueden alimentar una lógica tanto cooperativa como represiva. Como nos indicaba uno de los responsables del centro de trabajo:

En la plataforma utilizamos muy a menudo este tipo de dispositivos de grabación de resultados con los que confeccionamos listas estadísticas, y otros como la 'doble escucha', ya que nos permiten revisar nuestras opciones en materia de gestión de personal, y al mismo tiempo redefinir los ejes de progresión y de profesionalización de nuestros consejeros en línea. (Encargado del departamento de personal)

El control del ritmo de producción se efectúa esencialmente a través de dispositivos de control de tipo técnico. La introducción en los centros de llamadas de tecnologías de tipo PABX (comúnmente denominados autoconmutadores) permite la gestión y distribución automática de las llamadas telefónicas entrantes entre los diferentes puestos de trabajo. Dichos conmutadores posibilitan al mismo tiempo la configuración de grupos de operadores a los que les corresponde una determinada fila virtual de espera: una vez que finali- za una llamada, la siguiente (en posición de espera) bascula hacia la posición que se haya liberado. Los autoconmutadores son verdaderos ordenadores: es a través de ellos que se programa por ejemplo el tiempo de espera entre dos llamadas.

El autoconmutador hace también posible, y esta función es importante, la creación de una red de centros de llamadas, lo que permite organizar una cierta división del trabajo entre ellos: de una parte encontramos las plataformas telefónicas objeto de nuestro estudio como PHONING. Estas plataformas aseguran la primera atención al cliente, o la atención de primer nivel, y están encargadas de responder en función de 4 escenarios preestablecidos (cambio de domicilio, facturación, problemas con el pago y problemas técnicos). Estas plataformas emplean a unos 40 trabajadores y aseguran 1127 horas de comunicación telefónica por semana, lo que equivale a más de 1400 llamadas por día y por agencia. Las llamadas tienen unos tres minutos y medio de duración; tres segundos después de finalizar la comunicación precedente, una breve señal sonora en el casco auricular indica al teleoperador que un nuevo cliente acaba de entrar en línea.

De otra parte, en el caso de demandas de parte del cliente que escapen al circuito estipulado por los escenarios, los consejeros deben transferir la comunicación hacia los consejeros profesionales situados en los Centros de Relación con la Clientela (CRC). Estos centros de llamada, de creación reciente, proporcionan a los usuarios informaciones más precisas, con relación a sus instalaciones o a sus cuentas. La política de la empresa persigue diferenciar aún más las atribuciones de estos dos tipos de centros de llamadas, a la vez que la clientela se ve cada vez más fragmentada: de un lado los centros de llamadas especializados (los CRC) y caracterizados por una mano de obra competente y un servicio personalizado y de calidad y de otro lado, centros de llamadas como PHONING para tratar pedidos estandardizables de gestión corriente y susceptibles de generar ganancias de productividad. En último lugar, dentro de esta red encontramos también centros de llamada subcontratados para responder la noche y los domingos, y cuya fuerza de trabajo es obviamente exterior a la empresa.

En PHONING, el sistema técnico no sólo interviene en la regulación del ritmo objetivo de producción; también deter- 
mina el ritmo y el contenido de trabajo en el interior de la llamada telefónica. El programa informático regula la discusión con el cliente en tanto que dispositivo normalizador: por ejemplo, algunas ventanas del programa de trabajo no son accesibles hasta que las ventanas precedentes no han sido rellenadas de modo correcto. La comunicación del teleoperador con el cliente se ve en consecuencia permanentemente perturbada por esta intromisión del sistema técnico, y las interrupciones del cliente por el teleoperador son bastante corrientes generándose muy a menudo situaciones como la siguiente:

Cliente: Buenos días, señora, la llamo a propósito de una factura de $\mathrm{X}$ a una dirección que ya no existe porque ya no vivimos en ella y sin embargo hace ya más de tres meses que yo he señalado que no vivimos más en...

Teleoperadora: ¿Puede darme sus referencias por favor?

Cliente: Pfff (suspiro) Euhh sí...

La actividad de los trabajadores es muy rutinaria y altamente estresante. La observación del trabajo en largas secuencias pone en evidencia la repetitividad de la actividad de trabajo. Lo que movilizan ante todo los teleoperadores son rutinas lingüísticas que, siendo objeto de formateo y de control (número de señales sonoras antes de responder, ritual de presentación...) se convierten en, por utilizar la acertada fórmula de J. Boutet (2001), "contestadores automáticos". La secuencia canónica es aproximadamente la que sigue:

Cliente: Les llamo para un problema de factura, etc.

Teleoperador: ¿Cuáles son sus referencias?

Cliente: (dicta sus referencias)

Teleoperador: (escribe las referencias indicadas y consulta el informe del cliente)

La exigencia de rentabilidad pasa pues por un control estricto del tiempo y una normatividad muy fuerte del contenido de los intercambios con el público. Lo que para la dirección de los centros de llamadas, y en particular para la dirección de PHONING es ahorro de tiempo y dinero, los teleoperadores lo perciben como una desvalorización de sus capacidades y de su inteligencia. Los trabajadores son bastante explícitos cuando se refieren a algunos aspectos como la repetitividad en su trabajo:
Yo creo que tengo capacidades para realizar un trabajo mucho mejor. En la plataforma activo el piloto automático (...). Cualquiera puede desarrollar este trabajo, no son necesarias grandes capacidades para repetir contenidos como un lorito. A veces los clientes me preguntan si soy un contestador automático, no, no soy un contestador, soy una persona. Es muy fuerte, a veces me siento verdaderamente inútil. (Teleoperadora, 23 años)

La sensación de "activar el piloto automático” es muy fuerte entre los trabajadores entrevistados:

Hay una parte dentro de tí que se activa automáticamente, como cuando te vistes por la mañana o cuando comes y tienes la cabeza puesta en otra cosa, o simplemente no piensas en nada. Eso es lo que me sucede en el trabajo: a veces me dejo llevar por esos mecanismos internos que me permiten repetir una misma frase, una misma entonación, como una máquina, para no tener que pensar en lo absurda que me siento haciendo lo que hago. (Teleconsejera, 43 años)

No parece que existan muchas dudas sobre el lugar ocupado por los teleoperadores en la planificación y la puesta en aplicación de la arquitectura del proceso de trabajo. En el caso concreto de PHONING, donde el objetivo es el aumento de la productividad a corto plazo, los efectos han sido una degradación evidente de las condiciones de trabajo de los teleoperadores cuando otras posibilidades existen como lo demuestra la existencia dentro de la misma compañía de los Centros de Relación con la Clientela. La organización del trabajo no observa a los trabajadores como sujetos activos, como bien escribe Andrea del Bono (2000), por lo tanto con objetivos y motivaciones, sino que instala en ellos una sensación de programación que los aleja de su condición de personas.

Así no es sorprendente que la tasa de absenteísmo alcance el 40\% del personal de nuestro centro de llamadas, unas cifras desconocidas desde los años setenta. La banalización de la enfermedad profesional nos parece también extremadamente preocupante: una joven teleconsejera de 23 años nos respondía del modo siguiente en uno de los cuestionarios que hicimos pasar en el centro a una pregunta relativa a la frecuencia de toma de medicamentos: "no señor, no tomo medicamentos porque hace poco tiempo que estoy en la compañía”. 
Los dispositivos de normalización del comportamiento: cómo canalizar su emotividad al servicio de los objetivos de la empresa

La actividad de los teleoperadores requiere de otro aspecto, y que deriva del hecho que la calidad del producto deriva inmediatamente de la manera de trabajar (FLICHY \& ZARIFIAN, 2003). Esto es, la actividad necesita de una cierta flexibilidad en el trato así que una cierta implicación del teleoperador en la resolución de los problemas del cliente. El cliente debe sentirse "estimado" en dicha relación (COUSIN, 2002).

La movilización de los teleoperadores en los centros de llamadas comporta pues una cierta interiorización de los objetivos y de las obligaciones: los organizadores no parecen sólo obsesionados por la necesidad de objetivar el tiempo y de racionalizar al máximo las conversaciones, de convertirlas en algo abstracto o virtual en pos de una mayor eficiencia del punto de vista económico y productivo. El objetivo es también la implicación del asalariado, de su subjetividad, para que haga un uso de su tiempo subjetivo en el trabajo, un uso de su propia emotividad y de sus competencias lo más adecuado posible desde el punto de vista de la empresa.

Liliana Segnini (2000) explica que la referencia a la Programación Neuro-Lingüística tiene un peso fundamental en los procesos de racionalización de las prestaciones de servicio bancario en el Brasil:

Todos los centros de servicio a distancia que hemos estudiado recurren a técnicas de programación neuro-lingüística. Los documentos de las empresas especializadas en este tipo de técnicas las presentan como 'un sistema científico que subdivide el comportamiento en fracciones de información y en secuencias de aprendizaje fácil con el objetivo de intensificar la capacidad de comunicación para conseguir los resultados predeterminados, es decir el establecimiento de una relación de empatía, de armonía y de confianza entre la operadora y el cliente.

Liliana Segnini se refiere explícitamente a un documento de este tipo, editado por el Centro de dinámica de PNL (Programación Neuro-Lingüística $)^{5}$, donde las relaciones de empatía y de confianza, caracterizadas como proceso de acompañamiento, están subdivididas en: acompañamiento emocional, acompañamiento hacia un acuerdo, acompañamiento en el tono y el ritmo de la voz, acompañamiento en el plano de los valores y las creencias, acompañamiento cultural, acompañamiento en el plano de la finalidad. Cada uno de estos acompañamientos siendo dirigido al establecimiento de un sincronismo con el cliente.

En el mismo sentido que la investigadora brasileña, debemos destacar el lugar cada vez más importante que los dispositivos de coaching están tomando en los centros de llamadas. En PHONING, existen varios tipos de coaching, según que se refieran a los procesos y productos, o a las técnicas de comunicación; y en función de que sean dirigidos por responsables de formación, o por la jerarquía inmediata. La frecuencia registrada en PHONING es de un coaching por mes y agente. En ellos, los responsables del coaching evalúan las prestaciones del teleoperador sobre la base de una grabación del propio asalariado, o a partir de una conversación en vivo que posteriormente será diseccionada. De cada reunión de coaching se definen cuatro ejes de mejora que pueden referirse tanto a aspectos relativos a las técnicas de comunicación como a la actitud adoptada en el trabajo.

El teleoperador se compromete a perfeccionar al menos dos de estos ejes de mejora para el siguiente coaching, donde dos nuevos ejes serán definidos. Las reuniones de coaching tienen un carácter programado, y los resultados obtenidos repercuten, al menos a priori, sobre las posibilidades de los trabajadores de acceder a puestos de mayor responsabilidad jerárquica dentro de la estructura de mercado interno de la compañía.

En el primer caso, los teleoperadores trabajan, con los responsables de formación, aspectos ligados al control de la conversación con el cliente. Dicho de otro modo, se trata de interiorizar los mecanismos que permitan controlar los elementos aleatorios de cada conversación que puedan perturbar el desarrollo estándar de la misma:

¿Por qué el cliente te ha llevado ahí? Porque no has seguido el plan. ¿Por qué no le has propuesto servicios? Porque no has seguido el plan. Entonces es lógico que te haya planteado otro tipo de preguntas, que tú te hayas perdido contestándolas, y que hayas olvidado lo más importante. (Responsable de formación PHONING)

Pareciera como si con la reiteración y la presión sobre los teleoperadores, éstos aca-
${ }^{5}$ La Programación Neurolinguistica nace a mediados de los 70 en California, en la Universidad de Santa Cruz, del estudio de dos norteamericanos John Grinder y Richard Bandler, que han buscado entender lo que hacían realmente los mejores comunicadores o terapeutas para obtener sus resultados. Según Bandler, “la PNL proporciona el modo de empleo del cerebro". Podemos definirla también como el estudio de la experiencia subjetiva de la persona. La PNL busca modelizar un savoirfaire particular, una habilidad o un tipo de excelencia, buscando ante todo el modelo antes que el por qué, de manera que dicha excelencia pueda ser adoptada como práctica por el mayor número de personas posibles. No es por tanto extraño que su dominio de aplicación se extienda hoy en día al mundo del trabajo. 
barán asumiendo los objetivos de la gerencia como propios. Veamos cómo lo explica una responsable de formación:

Por ejemplo, esta mañana le hice el coach a una persona. (...) Una de las acciones estaba basada en la conformidad con las reglas cívicas mínimas: 'Le agradecemos su llamada, señor tal, y no dude en volvernos a llamar en caso de necesidad, gracias y hasta luego'. Entonces ella me dijo 'ya está, ya está hecho, ya forma parte de las costumbres', yo verifiqué, efectivamente había integrado el modo de funcionamiento de la manera adecuada y ya podíamos concentrarnos en otra acción. 'De hecho, es eso, le dije yo, tiene que integrarse en las costumbres, tiene que volverse un automatismo. Los principios claves del plan deben volverse automatismos. Una vez que eso es así, se ha ganado muchísimo en términos de calidad. No se puede sino ganar por esa vía'.

El carácter automatizante que busca imprimir el coaching en el comportamiento de los teleoperadores no escapa en absoluto al entendimiento de los propios trabajadores que entrevistamos:

El propósito que ellos tienen está muy claro: que no seamos personas, que respondamos como si fuéramos una máquina, un contestador automático. Y el coaching entra en la misma dinámica, olvidarte de quién eres y funcionar en base a expresiones hechas. Cuando en casa me sale el mismo tonito, me pregunto si no me voy a volver loca. (Consejera de clientela, 43 años)

Si este primer tipo de coaching subraya el carácter automatizante del trabajo desde la directividad en cuanto al respeto de las normas prescritas, la segunda dimensión insiste por el contrario en la reactividad en el momento de la comunicación con el cliente. Varios ejercicios de coaching insisten en la construcción de comportamiento típico por parte de los teleconsejeros:

Trabajamos mucho sobre la actitud que hay que tener al teléfono. La frase 'la sonrisa se escucha' no es ninguna estupidez. $\mathrm{Al}$ contrario, es algo fundamental. (...) En uno de los ejercicios que les hacemos hacer en la Escuela Clientela, les enseñamos a ver cómo por el tono de su voz pueden influir en el contexto emocional de una conversación. De hecho, la voz es una herramienta muy poderosa para producir sentimientos variados, si se sabe utilizar bien. En la escuela se ponen por grupos de cinco o seis, y les pedimos repetir la misma cosa, no sé, una presentación, ofrecer un producto...pero utilizando tonos diferentes de voz. (Cuadro de formación de la escuela clientela)
Esta misma responsable de formación nos habría de decir un poco más tarde en la entrevista: "comunicar bien no es más que seducir a quien te escucha”. Las técnicas de seducción ocupan un lugar primordial en la formación de los trabajadores: "influir el contexto emocional" y "producir sentimientos contrastados", y todo esto, ante la ausencia de contacto físico o visual, por la modulación de la voz.

Uno de estos ejercicios insistía por ejemplo en mostrar a los asalariados el poder de la simulación. Durante el ejercicio, una parte de los trabajadores circula con los responsables de la formación en torno a una sala mientras los otros, por parejas, simulan mantener conversaciones con el cliente. Aquellos que circulan deben ir anotando en una libreta aspectos referidos a la entonación, al contenido de los intercambios que van observando, mientras con la ayuda de los responsables se cuestionan sobre el mejor modo de construir la discusión con el cliente en función de las diversas situaciones posibles. Según un responsable que había participado en el ejercicio, a los asalariados les parecía divertido ver cómo, por une efecto de mimetismo, algunos acababan copiando ciertos gestos físicos y expresivos de otros teleoperadores, lo que según el mismo responsable "constituye el mejor modo de aprender”.

A través de este ejercicio y otros parecidos, los trabajadores deben aprender a "construir la relación” con el cliente, a través de técnicas preestablecidas de control de la conversación, incluyendo las técnicas de seducción que indicamos. Sin embargo, si durante los ejercicios de coaching lo que importa es la relación con el cliente y la calidad de la conversación, en situación de trabajo no es sino la cantidad de llamadas lo que verdaderamente importa a los organizadores. Dicho de otro modo: la actividad requiere un cierto tipo de "actitud" de la parte de los trabajadores, pero esta actitud se encuentra modelizada por los dispositivos puestos en práctica. Se puede por tanto afirmar que el coaching viene siendo utilizado como dispositivo de control suplementario, disfrazado como dispositivo formativo.

Proponemos entender la norma que impera en la construcción del comercial telefónico con la imagen del robot de seducción, que completa quizás la imagen propuesta por Gramsci (1949) de gorila amaestrado, y que resulta de la combinación inédita de modalidades de control: la 
brutalidad de trabajadores deshumanizados que deben ser capaces de activar algunas de sus capacidades de sociabilidad desde el autocontrol de su emotividad. Toda la paradoja organizacional está aquí presente y reposa sobre las espaldas de los teleoperadores.

En situación de trabajo, los trabajadores ejercen un trabajo sobre su emotividad, tal y como éste ha sido conceptualizado por Hochschild (1983). Este trabajo debe permitir la abstracción de los sentimientos privados, y la adaptación de estos mismos sentimientos privados a las normas y valores dispuestos por la empresa. Se trata de crear un personaje en el trabajo, lo que im- plica necesariamente una disociación. Dicha disociación no es neutra. El coaching daría las reglas generales de este personaje, el perfil tipo, así que facilitaría el proceso de adaptación, de adecuación al mismo perfil tipo, por medio del seguimiento sistematizado y metódico del proceso de profesionalización de cada trabajador. Algunos trabajadores lo han ilustrado de manera poderosa:

Aquí en la plataforma hay varias personalidades, pero ellos intentan modelarlas todas a partir de un patrón único, el comercial X. Como robots. Y presionan y presionan para que seamos todos iguales. (Consejera de clientela, 35 años)

\section{La subjetividad de los trabajadores como espacio de racionalidades en disputa: la tensión entre interactividad, ética y racionalización}

Los trabajadores de los centros de llamadas son plenamente conscientes que el coaching sirve para normativizar su comportamiento, para obligarles a desdoblarse, a jugar un rol. La manera en que los trabajadores de las plataformas movilizan o no, sus recursos en relación a la norma subjetiva, deviene pues significativa.

Lo que se requiere de los trabajadores de la mayor parte de espacios productivos es que sean capaces de mostrarse reactivos, disponibles, flexibles, móviles. Desde el punto de vista subjetivo, se les exige que acepten los criterios que rigen su actividad, así como una cierta lógica de comportamiento. La llamada a la implicación, sin embargo, no sustituye sino que se acompaña de una nueva normativización, de una nueva tecnicización, de los procesos de trabajo. Las nuevas formas de autonomía con todos los calificativos que han sido hasta ahora identificados se inscriben de este modo en los criterios de rentabilidad y de productividad de la empresa. La noción de combinatoria de modalidades de control que desarrollamos en este artículo pretende dar cuenta de dichos procesos. En el marco de los centros de llamada, es la persona del trabajador la que se encuentra comprometida en la actividad, condicionada por la yuxtaposición de imperativos cualitativos y cuantitativos que debe ser capaz de conciliar en situación de trabajo.

En este contexto, el objetivo de dispositivos como el coaching no es otro que el de hacer adoptar a los trabajadores una lógica de comportamiento respetuosa de los obje- tivos de rentabilidad de la compañía. Sin embargo, estos objetivos pueden desvelarse contradictorios con la "calidad de la prestación” o con la noción misma de servicio prestado al usuario, tal y como éstas vienen siendo comprendidas por los trabajadores mismos. Las quejas que hemos podido recensar durante nuestra inmersión de campo en relación a estos aspectos son numerosas:

Estamos ante el cliente, obligados de respetar los objetivos de rentabilidad de la empresa, no hay posibilidad de oponerse. En realidad estamos aquí para cortar al cliente de la compañía et no al revés, en realidad cabría preguntar si prestamos verdaderamente un servicio. (Consejera de clientela, 48 años)

Lo más duro no es solamente el tipo de trabajo que realizamos aquí, fatigoso, aburrido, estresante... lo más duro es tener que sonreír todo el tiempo, lo más duro es tener que 'tragar' la estrategia de la compañía, engañar al usuario, venderle servicios, cuando tú sabes que no lo necesita, lo más duro es participar de esa mentira. (Consejero de clientela, 42 años)

Las subjetividades de los trabajadores de este tipo de plataforma telefónica se encuentran doblemente afectadas. En primer lugar, porque la actividad es de naturaleza heterónoma, repetitiva y estresante, y no deja lugar a la creatividad y la autonomía de los trabajadores. En segundo lugar, porque no hay disociación de la personalidad que no sea patológica.

En situación de trabajo, los asalariados deben olvidar quiénes son, cuáles son sus 
emociones, su historia, su ética, sus motivaciones, para actuar en la representación de un personaje: el comercial sonriente. Esta representación, interiorizada punto por punto gracias a los dispositivos de coaching, es en realidad un aprentizaje de la disociación que obviamente no es neutra: la disociación implica la aceptación de criterios que rigen la finalidad de la acción, y que no son otros que los objetivos impuestos por la restructuración productiva de la compañía en un contexto neoliberal: productividad, rentabilidad, mercado.

La nueva orientación patronal busca asociar a los trabajadores a la nueva fase estratégica del desarrollo del capital, al nuevo proyecto de empresa; sin embargo, esta verdadera "revolución cultural" implica que los trabajadores rompan con una historia común, con una serie de principios éticos que gobernaron su actividad, con una cierta conciencia profesional construída en torno a la calidad del servicio. En cada acto de trabajo, esta dimensión entra en juego y estructura una línea de tensión, de oposición, entre los trabajadores y sus direcciones: la cuestión de la finalidad del trabajo.

En el contexto híbrido de los centros de llamada, hemos identificado dos tipos de prácticas de adaptación-resistencia en el trabajo, que no pueden comprenderse como polos confrontados o contradictorios. Al contrario, lo más sorprendente es que los trabajadores pueden bascular de las unas a las otras en el espacio de una sola llamada telefónica: cabría situarlas pues en un mismo continuum de prácticas que van del respeto estricto de la normas de trabajo a la implicación ética en la actividad. La misma línea de oposición (la finalidad del trabajo) está presente en los dos tipos de prácticas, y sin embargo sus horizontes son radicalemente divergentes.

Uno de los aspectos que más nos había sorprendido de la actividad de los consejeros es su capacidad para hacer otras cosas, hablar, reír, con otros compañeros, al tiempo que mantienen una discusión con el cliente. En algunas ocasiones es pues la pasividad, la distanciación del cliente por medio de la derisión, la "emotividad de superficie”, el recurso utilizado por los teleoperadores para resistir el estrés, la penosidad de la actividad, la agresividad del cliente (JEANTET, 2001). La emotividad de superficie corresponde a las ocasiones en que el teleoperador repite con el mismo tono altisonante y cantarín, un tono absolutamente forzado, como exagerando la norma de seducción requerida, los escenarios que le son impuestos: esas actitudes nos recuerdan a la función de salvapantallas de los computadores modernos cuando no se les activa ninguna tecla durante un tiempo determinado.

La fuerza coactiva y limitadora del sistema se ve en todo su esplendor; un sistema, como dice Del Bono, en el que no se concibe a los seres humanos como lo que son, sino como meros apéndices de un proceso. Estas actitudes corresponden en este contexto a verdaderas líneas de fuga en la representación del personaje, en la simulación del espectáculo, pero acaban siendo muy penosas para los trabajadores de los centros, y extremadamente desvalorizantes. En este tipo de actitud se manifiesta en el rechazo por parte del teleconsejero de su actividad, y como escribe Angel Lara:

la separación entre fuerza del trabajo y sentido de la actividad es máximo, llevando en muchos casos al abandono del empleo. (LARA, 2004, p. 46)

No son éstas sin embargo las únicas formas de resistencia que hemos identificado en los centros de llamadas. En diferentes situaciones de trabajo hemos visto cómo los teleconsejeros pueden controlar sus emociones y activar algunas de sus capacidades de sociabilidad como si se tratara de un don.

Sería por ejemplo el caso de los trabajadores que continúan dando su número de teléfono de puesto para construir una relación más personalizada con un cliente, porque su expediente parezca demasiado complicado, o porque la persona misma requiere una atención particular. Los trabajadores se informan entre ellos sobre cuáles son los problemas, nuevos o viejos, que van apareciendo para el desarrollo de la actividad, o sobre cómo responder a cierto tipo de cuestionamiento que no aparece necesariamente en los guiones. Los teleconsejeros constituyen de modo informal una serie de indicadores de riesgo que utilizan para evitar transferir la llamada a los CRC profesionales, por lo que su actuación debe entenderse en términos de autovalorización de su propia actividad. La práctica de la personalización contraviene completamente las indicaciones de la jerarquía a este respecto, y las notas informativas y las reuniones para erradicar este tipo de prácticas colectivas son bastante corrientes. En una de esas reuniones a las que pude asistir los consejeros objetaban a la jerarquía 
que las situaciones deben ser tratadas caso por caso, en función de las necesidades particulares de cada persona; mientras que la dirección subrayaba por su parte que la personalización dificultaba el funcionamiento correcto de la red. También objetaban que con esas prácticas se oculta el número total de llamadas. En situación de trabajo el colectivo persiste en este tipo de prácticas, y es interesante constatar que la jerarquía inmediata cierra los ojos y protege al colectivo, ya que al fin y al cabo todo el mundo sale beneficiado de este tipo de funcionamiento colectivo.

En esta situación estamos confrontados a una oposición fuerte entre dos maneras de entender la noción de calidad de la prestación, y más en general, de la representación que unos y otros se hacen del cliente. Dicho de otro modo, situaciones como éstas podrían servir de fundamento para la afirmación de una conciencia profesional alternativa, para la contestación de los principios de base sobre los que reposa la definición de competencia, de jerarquía, resumiendo, para la creación de un colectivo de trabajo. La mayor parte de los teleoperadores rechazaba por ejemplo proponer servicios pagos a clientes que llaman porque tienen problemas para pagar sus facturas:

Es que es una bestialidad que te obliguen a vender un servicio a alguien que no tiene con qué pagarte. ¿Con qué cara le digo yo a una persona en esa situación que tenemos un servicio maravilloso que le va a solucionar todos sus problemas? (Teleconsejera, 27 años)

En otras ocasiones, los teleconsejeros se extralimitan en sus atribuciones formales al tomar partido por un tipo de servicio antes que por otro, por ejemplo el servicio de una compañía que consideran mejor que el de otra:

Un ejemplo de eso lo tienes cuando nos preguntan si es mejor el gas o la electri- cidad. La dirección nos obliga a no responder a ese tipo de pregunta y a enviar al cliente a los CRC de ambas compañías, que desde el proceso de privatización que sufrimos están separadas, y que luego el mismo cliente decida. Pero es que es absurdo, está clarísimo que el gas es mejor que la electricidad, porque es más barato y más limpio, así que no dudamos en decirle al cliente que escoja el gas antes que la electricidad. Y pregúntale a cualquiera de nosotros, todos lo hacemos igual (Teleconsejera, 32 años)

Cabe destacar que estas nuevas formas de resistencia pueden estar a la base de colectivos de trabajos renovados y potencialmente críticos con los valores y las condiciones de realización del trabajo de las empresas donde están empleados. Así, no en el caso de PHONING sino en otro centro de llamadas que visitamos, los teleoperadores se declararon en huelga porque el producto estrella de la compañía a la que pertenecen, un artículo que permite la conexión simultánea a la televisión, internet y a la red telefónica tradicional, no funcionaba correctamente. Los teleoperadores justificaron su movilización por lo insoportable que se había vuelto su trabajo, el contacto cotidiano con unos clientes que llamaban coléricos contra la compañía porque obviamente se sentían estafados; al tiempo, la compañía exhortó a los teleoperadores a seguir vendiendo el producto como si nada sucediera, algo a lo que los teleoperadores oposieron: "no podemos seguir mintiendo a los clientes como hasta ahora" (CALDERÓN, 2006).

En estos casos que podríamos denominar de resistencia activa, sería pues paradójicamente la implicación subjetiva en la actividad, con todo lo que ello conlleva, que permite a los teleconsejeros la creación de un espacio de autovalorización y de rearticulación de solidaridades, de crítica a la racionalidad de la organización.

\section{Conclusión o apuntes por una toma en consideración del trabajo como valor de uso}

Los centros de llamadas representan el espacio de una interesante contradicción, reveladora de evoluciones de más largo alcance en el mundo del trabajo. Nunca antes en la historia de la racionalización, el "factor humano" ha ocupado un lugar tan decisivo para asegurar la valorización del capital: lo que cuenta hoy en día no es sólo el tiempo objetivo de producción, sino el tiempo subjetivo de producción. A la rivalidad (tayloriana) en torno al primero debería dar paso a un uso conjunto del segundo (entre organizadores y asalariados) para conseguir los mismos objetivos. La radicalidad del cambio se ve sin embargo limitada, al menos en los centros de llama- 
das, por la existencia de dos variables: de una parte, estos objetivos no son negociables, y de otra parte, el predominio de las normas temporales y del marco prescriptivo demuestran el vigor de la inspiración tayloriana en este tipo de organización.

Los centros de llamada representan así la evolución quizás paradigmática del taylorismo hacia una nueva organización de tipo neo-taylorista que requiere, a la diferencia de las organizaciones estrictamente taylorianas, una cierta implicación subjetiva de parte de los trabajadores; siendo esta implicación modulada, lo hemos mostrado, por los dispositivos de formación puestos en aplicación. Sin embargo, a partir del momento en el que el tiempo subjetivo de producción entra en la línea de mira del organizador, este mismo tiempo subjetivo deviene el espacio de una tensión que se estructura a partir de los registros en los que se sustenta la práctica de trabajo, puesto que es la finalidad misma del trabajo que viene a ser comprometida: ¿por qué los trabajadores trabajan? ¿por qué se implican en la resolución de los problemas del cliente, buscando siempre nuevos resquicios en los que hacer vivir su compromiso? Estos dos cuestionamientos reflejan quizás una nueva dimensión de la relación capitaltrabajo; una dimensión que no debe entenderse de modo abstracto sino que está muy presente, cada día, en lo más concreto de la actividad de los trabajadores, en cada acto de trabajo, en tanto que organizadora si se quiere de la acción en torno a al menos dos lógicas confrontadas.

Una teleconsejera de 23 años me comentaba en una de las entrevistas que tuve la oportunidad de realizar:

A todos nos piden que mintamos un poco, que tampoco es tan grave, que nos olvidemos de lo que verdaderamente pensamos, pero al final tantas pequeñas mentiras se acaban convirtiendo en una gran mentira y todos somos partícipes de esa gran mentira.

Obviamente se podría considerar que el sistema funciona porque los trabajadores colaboran con la mentira, con la injusticia. Sin embargo, y de modo igualmente también manifiesto, el sistema funciona sólo porque al mismo tiempo cada uno le opone una resistencia, porque los trabajadores, lejos de comportarse como simples apéndices de la máquina, ponen un poco de su humanidad en la lógica ciega del sistema. En este punto, cabe suponer que cada trabajador hace trampas en función de sus propias exigencias éticas y se esfuerza por subvertir las relaciones sociales de dominación y de explotación que pesan sobre él en función de esas mismas exigencias. Pensar la cuestión del cambio implica por tanto no olvidar que lo que da carne y vida al sistema es tanto la sumisión como esta misma resistencia a menudo menospreciada o minusvalorada por los propios científicos del trabajo. Basta volver la vista a las conceptualizaciones aún predominantes del trabajo situadas en la tradición de Aristóteles, Arendt o Habermas: sólo la racionalidad comunicacional, nunca la instrumental (de la que hace parte el trabajo), es portadora de perspectivas de emancipación.

Y sin embargo, la escucha de los trabajadores que han basculado hacia la enfermedad muestra bien que, en la mayor parte de los casos, las contradicciones de la actividad son tomadas muy en serio, y que los trabajadores no están dispuestos a asumir cualquier vía de manera impensada para alcanzar el objetivo prefijado. Lo que los trabajadores, y en particular los trabajadores de los centros de llamadas, comprometen en la actividad es algo más que la satisfacción de los objetivos necesariamente abstractos fijados por la empresa.

Las direcciones de las empresas, lo decíamos antes, han hecho penetrar el mercado mundial hasta el puesto de trabajo: los imperativos financieros y productivos al más corto plazo, el ajustamiento constante e immediato a las variaciones de la demanda con sus corolarios de diversificación y flexibilización y podríamos añadir un largo etcétera. Sin embargo, la sociedad como sistema de normas y de valores irreductibles a los valores del mercado, también está presente en el puesto de trabajo, en cuanto que incorporada a los cuerpos y a la subjetividad de los agentes. De esta manera, las tensiones entre normas mercantiles y normas sociales, y por tanto de las normas políticas más generales, se viven en sus dimensiones más concretas en el corazón mismo de la actividad.

Las direcciones rechazan sistemáticamente establecer un arbitrio entre dichas normas, porque no les corresponde o porque no les conviene. Sin embargo, los trabajadores, y más particularmente los teleoperadores, no pueden rechazar dicho arbitrio, ya que su actividad les impone justamente establecer una mediación cotidiana entre las leyes del mercado y las leyes sociales más básicas como el respeto del otro y la sinceridad. Este arbitrio los trabajadores lo 
realizan, como no podría ser de otra manera, con sus subjetividades, sus historias y sus valores, y les lleva, a menudo, al fracaso, al sufrimiento y a la enfermedad, pero también al éxito, a la invención, a la experiencia y al colectivo, es decir, al reconocimiento de sí mismo en los demás.

Manifiestamente, el principal problema es que todo esto parece permanecer constricto al ámbito individual, y en mayor parte impensado, naturalizado en tanto que no reconocido, en la mayor parte de las situaciones. Las prácticas de trabajo basculan así del estricto respeto de las consignas a la implicación activa en los problemas

\section{Referencias bibliográficas}

ANTUNES, R. Os sentidos do trabalho. São Paulo: Boitempo, 1999.

BOLTANSKI, L.; CHIAPELLO, E. Le nouvel esprit du capitalisme. Paris: Gallimard, 1999. (NRF essays)

BONO, A. del. Call Centers, ¿el trabajo del futuro? El caso de estrategias telefónicas S.A. (Estratel). Sociología del Trabajo, n. 39, p. 3-31, 2000.

Call centers, estrategias de flexibilidad y nuevas experiencias laborales. In: CASTILLO, J. J. El trabajo recobrado. Madrid: Miño y Dávila, 2005.

BOUTET, J. Le travail devient-il intellectuel? Travailler, n. 6, p. 55-70, 2001.

BUSCATTO, M. Les Centres d'appels, usines modernes? Les rationalisations paradoxales de la relation téléphonique. Sociol. trav., v. 44, n. 1, p. 99-117, 2002.

CALDERON, J. L'implication quotidienne dans un centre d'appel. Les nouvelles 'initiatives éducatives. Travailler, n. 13, p. 7594, 2005.

Repensar la cuestión de la resistencia en el trabajo o buscando al trabajador perdido. Sociología del Trabajo, n. 56, 2006.

CALLAGHAN, G.; THOMPSON, P. We recruit attitude: the selection and shaping of routine call centre labour. Journal of Management Studies, v. 39, n. 2, p. 233-254, 2002.

CASTILLO, J.-J. Sociología del trabajo: un proyecto docente. Madrid: CIS-SXXI, 1996.

COSSALTER, C. La compression des temps. Quand les services financiers s'équipent en del usuario, poniendo pues en grave peligro el principio de universalidad que está en la base misma de la noción de servicio público.

Desde el punto de vista de los trabajadores, está por ver si la estabilización y la reproducción de las prácticas de resistencia de tipo activo permiten reequilibrar las relaciones de fuerza (colectivos de trabajo que toman la mano sobre la 'profesionalización de la actividad'), o si es por el contrario el sentimiento de desvalorización de la actividad, que es forzosamente una desvalorización de sí mismo, el que se acaba imponiendo.

plateaux téléphoniques. In: LINHART, D.; MOUTET, A. Le travail nous est compté. France: La Découverte, 2005. (Collection Recherches)

COUSIN, O. Les ambivalences du travail. Les salariés peu qualifiés dans les centres d'appel. Sociol. trav., v. 44, n. 4, p. 499-520, 2002.

DUFAU, M.; STUCHLIK, J-B. L'organisation du travail dans les centres d'appel. Lyon: Anact, 2002.

DURAND, J.-P. La chaîne invisible. Flux tendu et servitude volontaire. Paris: Le Seuil, 2004.

FLICHY, P.; ZARIFIAN, P. Les centres d'appel, Réseaux, v. 20, n. 114, 2003.

GADREY, J. La productivité en question. Paris: Desclée de Brouwer, 1996.

GRAMSCI, A. Note sul Machiavelli, sulla politica e sullo Stato moderno. Torino: Einaudi, 1949.

HABERMAS, J. The theory of communicative action: "reason and the rationalization of society". London: Heinemann, 1984. v. 1.

HOCHSCHILD, A. The managed heart: the commercialization of human feeling. Berkeley: University of California Press, 1983.

JEANTET, A. La relation sociale de service. 2001. Thèse (Sociologie), Université Paris X-Nanterre, Paris, 2001.

LARA, A. El telemarketing en España: materiales para una cartografía del mundo del trabajo contemporáneo. Sociología del Trabajo, n. 49, p. 27-59, 2004. 
LECHAT, L.; DELAUNAY, J. C. Les centres d'appels: un secteur en clair-obscur. Paris: L'Harmattan, 2003.

LINHART, D.; MOUTET, A. Le temps nous est compté. Paris: La Découverte, 2005.
SEGNINI L. Le télémarketing: un vrai travail moderne. Cahiers du Genre, n. 28, p. 117-131, 2000.

VELTZ, P.; ZARIFIAN, P. Vers des nouveaux modèles d'organisation. Sociol. trav., v. 35, n. 1, p. 112-139, 1993. 